\title{
A minimally altruistic refinement of Nash equilibrium
}

\author{
Emin Karagözoğlu*, Kerim Keskin, Çağrı Sağlam \\ Bilkent University, Department of Economics, 06800 Bilkent, Ankara, Turkey
}

\section{H I G H L I G H T S}

- A rather minimalist notion of altruism is introduced.

- It is based on a lexicographic preference structure.

- Minimal altruism is used to refine the set of Nash equilibria in normal form games.

- Three independent existence proofs are provided for this new refinement concept.

- An in-depth sensitivity analysis is conducted and expository examples are given.

\section{A R T I C L E I N F O}

\section{Article history:}

Received 18 June 2013

Received in revised form

7 October 2013

Accepted 9 October 2013

Available online 16 October 2013

\begin{abstract}
A B S T R A C T
We introduce a minimal notion of altruism and use it to refine Nash equilibria in normal form games. We provide three independent existence proofs, relate minimally altruistic Nash equilibrium to other equilibrium concepts, conduct an in-depth sensitivity analysis, and provide examples where minimally altruistic Nash equilibrium leads to improved predictions.
\end{abstract}

(c) 2013 Elsevier B.V. All rights reserved.

\section{Introduction}

In the last thirty years, we witnessed a surge in experimental studies in economics reporting altruistic or other-regarding behavior (see Güth et al. (1982), Roth et al. (1991), Forsythe et al. (1994), Güth and Van Damme (1998), Fehr and Gächter (2002) and Charness and Rabin (2002) among others). This observation is in stark contrast with the selfish man, an assumption to which most theoretical models in economics, if not all, were implicitly or explicitly subscribed. Nevertheless, the experimental evidence in favor of other-regarding behavior is so overwhelming that we also see an increasing number of theoretical models explaining/incorporating altruism (see Rabin (1993), Levine (1998), Fehr and Schmidt (1999), Bolton and Ockenfels (2000), Gintis et al. (2003), Fehr and Fischbacher (2003), Falk and Fischbacher (2006) and Cox et al. (2007, 2008) among others).

With a few exceptions (see Cox et al. (2008)), in most theoretical papers modeling other-regarding behavior, altruism is incorporated into their models with an additively separable utility function: an agent directly cares about others through altruism or indirectly cares about others through his inequality aversion. In this paper, we introduce a different notion of altruism and use it to refine Nash equilibria in normal form games. In modeling otherregarding behavior, we use agents' preferences as a work-horse rather than their utility functions. In particular, we assume that each agent may care about the well-being of a set of other agents

\footnotetext{
* Corresponding author. Tel.: +90 3122901955; fax: +90 3122665140.

E-mail addresses: karagozoglu@bilkent.edu.tr, eminkaragozoglu@gmail.com (E. Karagözoğlu).
}

in addition to his own well-being, in a lexicographic fashion: a set of agents (including the agent himself) each agent cares about and an order on these agents are defined, where an agent's own wellbeing is at the top of this priority order. Agents may best respond to others' strategies with lexicographic preferences on outcomes. Therefore, an agent in a strategic game first maximizes his own well-being and then among the set of outcomes that maximize his own well-being (i.e., his best-response set), he prefers the ones that maximize the well-being of the agent ranked second in his priority order and so on. ${ }^{1}$ Clearly, this notion of altruism is much less demanding than the standard notion of altruism where all agents' utilities/payoffs enter into a utility function at the same level but possibly with varying weights. This is why we label it as minimal altruism. $^{2}$ If an agent's priority set is a singleton, then this means

\footnotetext{
1 A step in a similar direction is taken by Dutta and Sen (2012) in the social choice context. These authors introduce partially honest individuals, who strictly prefer telling the truth if doing so does not lead to an outcome worse than lying does. The presence of such individuals turns out to be crucial for obtaining Nash implementability. Similar minimal or costless honesty notions are also used by Laslier and Weibull (2013) and Dutta and Laslier (2010) in jury and voting contexts, respectively. Finally, Doğan (2013) introduce responsible agents who first care about their own utility and then social efficiency (in a lexicographic manner) in an allocation problem.

2 Our minimal altruism notion is different and even less demanding than the notion Fishkin (1982) introduced: Fishkin's principle of minimal altruism, as a mora principle, stipulates that if an agent, by incurring minor personal costs, can bring about great benefits (or prevent great harm), then he/she is morally obligated to do so. It is also compatible with the limits of altruism Hardin (1977) put forward: "Never ask a person to act against his own self-interest". Finally and more closely, it is identical to the interdependence condition, minimal altruism, formulated by Knoblauch (2001).
} 
he only cares about himself and thus he is a selfish agent. If his set is not a singleton, then he is a minimally altruistic agent. ${ }^{3}$ Note that an agent's priority set does not have to include all other agents in this case: he may only care about the well-being of a subset of others in which case he is still considered to be a minimally altruistic agent. This case would also resemble nepotism.

The minimal altruism we introduce is more relevant (or useful) in situations where (i) agents cannot influence their own payoffs to a great extent but can still influence others' payoffs, (ii) agents are indifferent between multiple actions (i.e., there are multiple actions/strategies that maximize their own well-being), or (iii) some agents in a strategic situation have just a single action. In many such circumstances, it is plausible to assume that most people would also care about others' well-being as well. In fact, experimental findings in Engelmann and Strobel (2004), Güth et al. (2010, 2012) and Cappelen et al. (2013) and in many other experiments using impartial spectators, provide - at least a partial - support for our notion: in these experiments, subjects whose earnings are (at least locally) fixed and not affected by their decisions make distributive decisions that are in line with efficiency, equality and equity concerns.

We provide three independent existence proofs for our equilibrium concept: using Kakutani's fixed point theorem as in the existence proof of Nash equilibrium, using Zhou's fixed point theorem as in the existence proof of Nash equilibrium in games with strategic complementarities, and using the existence of Berge-Nash equilibrium. The first existence result is the most natural and standard one since Nash equilibrium is a special case in our setup. With adaptations of some of the sufficient conditions for the existence of Nash equilibrium and an additional condition (level- $k$ empathy), we guarantee the existence of minimally altruistic Nash equilibrium. On the other hand, the reason why we resort to games with strategic complementarities is that our refinement has much to offer when the game has more coordination aspect than pure competition aspect and when players' potential to influence others' payoffs is substantial. Games with strategic complementarities satisfy these requirements to a great extent. Finally, we use Berge-Nash equilibrium for yet another existence result. The Berge equilibrium concept assumes a different - and a rather extreme sort of altruistic behavior. Thus, it is reasonable to investigate the relationship between minimally altruistic Nash equilibrium and Berge-Nash equilibrium.

Our results show that minimally altruistic Nash equilibrium leads to better and sharper predictions than Nash equilibrium in many instances, if one believes that our notion of altruism is realistic. As a result of the richer structure of game definition that incorporates priority sets and priority orders, even players who have single strategies or players who are indifferent between all of their strategies can influence the set of minimally altruistic Nash equilibria, which is a feature of many real-life circumstances. Note that such players cannot influence the set of Nash equilibria. On the other hand, it is also important to emphasize that the minimally altruistic refinement operates on the set of individual best responses and not on the set of Nash equilibria. ${ }^{4}$ This has

\footnotetext{
3 Mathematically speaking, modeling altruism in this fashion (i.e., with lexicographic preferences) is equivalent to taking the limit of CES family of utility functions (over agents' utilities), where the weight an agent attaches to an agent ranked at $k$ th place in his priority order becomes infinitely greater than the weight he attaches to an agent ranked at $(k+1)$ th place. Since all agents rank themselves at the top of their priority order, they care about themselves infinitely more than any other agent. Hence, the adjective, minimal, also makes sense from a mathematical point of view.

4 First of all, refining the set of Nash equilibria by directly eliminating some of them would not be a significant innovation from an intellectual perspective. Moreover, what is modeled in that case would not be pure altruism since otherregarding behavior would not influence players' behavior in the game.
}

important and interesting implications: even though players care about others' well-being only after maximizing their own wellbeing, this does not necessarily imply that they will be equally well off in cases where they are selfish and altruistic. For instance, a player who starts to care about others (or becomes selfish) may face a less or more favorable set of payoffs by doing that. Similarly, a player who nobody cared about before may face a less favorable set of payoffs after some (or even all) players start caring about him. Concerning the comparison between minimally altruistic Nash equilibrium and Berge-Nash equilibrium, we show that in many instances where Berge-Nash equilibrium does not exist, minimally altruistic Nash equilibrium exists. Finally, by conducting an indepth sensitivity analysis we show that the set of equilibria is highly sensitive to the set of players each player cares about and the priority order each player has.

It is worth mentioning that minimally altruistic refinement is a complement rather than a substitute for other refinement concepts (e.g., essential equilibrium etc.). Our refinement is different than these in that it is not based on players making mistakes and hence does not use any perturbations. Moreover, minimally altruistic refinement is also different from coalition-proof and strong Nash refinements in that it is not based on a coalitional structure. Nevertheless, we do not see any element in the minimally altruistic refinement, which would pose a problem for applying it together with one of these other refinements.

The paper is structured as follows: in Section 2, we present some preliminaries, i.e., definitions and results we employ throughout the paper. In Section 3, we introduce minimal altruism, provide the formal definition for minimally altruistic Nash equilibrium and existence results. Section 4 provides further results. In Section 5, we present some examples where we refine the set of Nash equilibria using minimal altruism. Finally, Section 6 concludes.

\section{Preliminaries}

In what follows, we provide some definitions and theorems that we utilize throughout the paper. First, a set of definitions:

Definition 1 (Quasiconcavity). A function $f: X \rightarrow \mathbb{R}$ on a convex set $X$ is quasiconcave if for every $a \in \mathbb{R},\{x \in X \mid f(x) \geq a\}$ is convex.

Definition 2 (Closed Graph). Let $X$ and $Y$ be any topological spaces. A correspondence $F: X \rightarrow Y$ has a closed graph if $x \in F(y)$ for any two sequences $\left(x^{n}\right) \rightarrow x$ and $\left(y^{n}\right) \rightarrow y$ with for every $n: x^{n} \in F\left(y^{n}\right)$.

Definition 3 (Upper Semi-Continuity). A function $f: X \rightarrow \mathbb{R}$ is upper semi-continuous if for every $x \in X$ and every sequence $\left(x^{n}\right)$ with $\left(x^{n}\right) \rightarrow x, \lim \sup f\left(x^{n}\right) \leq f(x)$.

Definition 4 (Lattice and Complete Lattice). A partially ordered set is a lattice if it contains the supremum and the infimum of all pairs of its elements. A lattice is complete if each nonempty subset has a supremum and an infimum.

Definition 5 (Subcomplete Sublattice). Let $X$ be a lattice and $Y \subset$ $X$ be a sublattice. $Y$ is a subcomplete sublattice of $X$ if, for each nonempty subset $Y^{\prime}$ of $Y, \bigvee_{X} Y^{\prime}$ and $\bigwedge_{X} Y^{\prime}$ exist and are contained in $Y$.

Definition 6 (Supermodularity). Let $X$ be a lattice. A function $f$ : $X \rightarrow \mathbb{R}$ is supermodular if for all $x, x^{\prime} \in X, f(x)+f\left(x^{\prime}\right) \leq f\left(x \wedge x^{\prime}\right)+$ $f\left(x \vee x^{\prime}\right)$. 
Definition 7 (Increasing Differences Property). Let $X$ be a lattice and $T$ be a partially ordered set. A function $f: X \times T \rightarrow \mathbb{R}$ has increasing differences in $(x, t)$ if $f\left(x, t^{\prime}\right)-f(x, t)$ is increasing in $x$ for every $t<t^{\prime}$.

Definition 8 (Veinott Increasingness). Let $X$ be a lattice. A correspondence $F: X \rightarrow X$ is Veinott-increasing if for each $x, y \in X$ with $x<y, a \in F(x)$ and $b \in F(y)$ implies $a \wedge b \in F(x)$ and $a \vee b \in F(y)$.

Definition 9 (Fixed Point). A fixed point of a function $f: X \rightarrow X$ is $x \in X$ such that $f(x)=x$. The set of fixed points of a correspondence $F: X \rightarrow X$ is defined as $\{x \in X \mid x \in F(x)\}$.

Finally, we refer to the following theorems throughout the paper:

Theorem 1 (Kakutani (1941)). Let $X$ be a nonempty, compact, and convex subset of Euclidean space. If $F: X \rightarrow X$ is a nonempty-valued and convex-valued correspondence with a closed graph, then $F$ has a fixed point.

Theorem 2 (Zhou (1994)). Let X be a nonempty, complete lattice and $F: X \rightarrow X$ be a nonempty-valued correspondence. If $F$ is Veinottincreasing and $F(x)$ is a subcomplete sublattice of $X$ for every $x \in X$, then the fixed point set of $F$ is a nonempty complete lattice.

Theorem 3 (Topkis (1998)). Let $X$ be a nonempty, complete lattice, $T$ be a partially ordered set, and $Y: X \times T \rightarrow X \times T$ be a correspondence. If $Y$ is increasing, and $Y(x, t)$ is a nonempty subcomplete sublattice of $X \times T$ for each $(x, t) \in X \times T$, then

(i) for all $t \in T$, there exists a greatest (least) fixed point of $Y(x, t)$,

(i) the greatest (least) fixed point of $Y(x, t)$ is increasing in $t$ on $T$.

\section{Minimally altruistic Nash equilibrium}

In this section, we first define minimal altruism and minimally altruistic Nash equilibrium concepts. Then, we show that minimally altruistic Nash equilibrium is indeed a refinement of Nash equilibrium. Finally, we provide existence results for this refinement concept.

\subsection{Minimal altruism and refinement}

Let $\Gamma=\left(N,\left(X_{i}\right)_{i \in N},\left(u_{i}\right)_{i \in N}\right)$ be a normal form game where $N$ is the finite set of players, $X_{i}$ is the set of strategies for player $i$, and $u_{i}$ is player $i$ 's payoff function. Let $S_{i}$ be a subset of $N$ that includes $i$ and $\left(S_{i}\right)_{i \in N}$ be a collection of such subsets for all $i \in N$. Then, let $\succ_{i}$ be a strict order defined on $S_{i}$ such that $i \succ_{i} j$, for all $j \in S_{i} \backslash\{i\}$. Finally, for any $i \in N$ and $j \in S_{i}$, let $\varphi(i, j)$ denote agent $j$ 's rank in agent $i$ 's priority order, $\succ_{i} .{ }^{5}$ We define $\Gamma_{M A}=$ $\left(N,\left(X_{i}\right)_{i \in N},\left(S_{i}\right)_{i \in N},\left(\succ_{i}\right)_{i \in N},\left(u_{i}\right)_{i \in N}\right)$ as a minimally altruistic version of a normal form game where each player is associated with one priority set and a priority order on it. To define the minimally altruistic Nash equilibrium, we first define minimal altruism.

Definition 10. Let $R_{i}$ be a preference relation on $\mathbb{R}^{|N|}$. Let $P_{i}$ denote the strict preference and $I_{i}$ denote the indifference induced by $R_{i}$. For a given $\left(S_{i}, \succ_{i}\right)$, an agent $i$ is a minimally altruistic agent if his

\footnotetext{
5 For the sake of completeness, for every $i, j \in N$ with $j \notin S_{i}$, we set $\varphi(i, j)=n+1$.
}

preference among any two payoff vectors $e=\left(e_{1}, \ldots, e_{i}, \ldots, e_{|N|}\right)$ and $e^{\prime}=\left(e_{1}^{\prime}, \ldots, e_{i}^{\prime}, \ldots, e_{|N|}^{\prime}\right)$ is written as

$e P_{i} e^{\prime} \quad$ if $e_{i}>e_{i}^{\prime}$

$e P_{i} e^{\prime} \quad$ if $e_{i}=e_{i}^{\prime} \quad$ and $\quad e_{j}>e_{j}^{\prime} \quad$ where $\varphi(i, j)=2$

......

$\ldots \ldots$

$e P_{i} e^{\prime} \quad$ if $\forall k \in S_{i} \backslash\{m\}, e_{k}=e_{k}^{\prime}$ and $e_{m}>e_{m}^{\prime}$ where $\varphi(i, m)=\left|S_{i}\right|$

$e I_{i} e^{\prime} \quad$ if $\forall k \in S_{i}, \quad e_{k}=e_{k}^{\prime}$.

Now, we can define minimally altruistic refinement of Nash equilibrium. For an agent $i \in N$ and for every $y \in X$, let

$X_{i, 1}(y)=\arg \max _{x \in X} u_{i}\left(x_{i}, y_{-i}\right)$.

Then, for every $y \in X$ and for every $k=1, \ldots,\left|S_{i}\right|-1$, let

$X_{i, k+1}(y)=\arg \max _{x \in X_{i, k}(y)} u_{j_{k+1}}\left(x_{i}, y_{-i}\right)$

such that $j_{1}=i$ and $\varphi\left(i, j_{k+1}\right)=k+1$.

Definition 11 (MANE). A strategy profile $x^{*} \in X$ is a minimally altruistic Nash equilibrium (MANE) if for all $i \in N: x^{*} \in X_{i,\left|S_{i}\right|}\left(x^{*}\right)$.

Notice that $S_{i}$ and $\succ_{i}$ are included in the definition of the game $\left(\Gamma_{M A}\right)$ whereas $R_{i}$ is included in the definition of the equilibrium concept. Thus, how the information provided by $S_{i}$ and $\succ_{i}$ is processed is given in the equilibrium concept. It can be argued that we follow a normative approach here, by including altruism in the equilibrium concept, MANE. Alternatively, one can follow a positive approach by investigating the Nash equilibrium outcomes in games where players have altruistic preferences. ${ }^{6}$ Finally, it is worthwhile emphasizing that minimally altruistic Nash equilibrium uses, whereas the Nash equilibrium concept ignores, the information provided by $S_{i}$ and $\succ_{i}$.

Notice that for any player $i, X_{i, k}(\cdot)$ in this definition is a subset of the best response correspondence of $i$ according to Nash, $B R_{i}(\cdot)$, which is equal to $X_{i, 1}(\cdot)$. This directly implies the following result.

Proposition 1. Minimally altruistic Nash equilibrium is a refinement of Nash equilibrium.

Proof. Take any MANE, $x^{*} \in X$. Then for all $i \in N, x^{*} \in X_{i,\left|S_{i}\right|}\left(x^{*}\right)$. By definition, for all $i \in N, x^{*} \in X_{i, 1}\left(x^{*}\right)=B R_{i}\left(x^{*}\right)$. Thus, $x^{*}$ is a $N E$.

The following example demonstrates that a $N E$ is not necessarily a MANE.

$$
\begin{array}{l|l} 
& x_{2} \\
x_{1} & 2,1 \\
y_{1} & 2,0 \\
\cline { 2 - 2 } &
\end{array}
$$

Here, both strategy profiles are $N E$, but $\left(y_{1}, x_{2}\right)$ is not a MANE given that $S_{1}=\{1,2\}$.

\subsection{Existence}

In the following parts, we provide three independent existence results: utilizing (i) Kakutani's fixed point theorem, (ii) Zhou's fixed point theorem, and (iii) the Berge-Nash equilibrium existence result. $^{7}$

\footnotetext{
6 We thank Tarık Kara for bringing up this issue during our discussions.

7 A mixed strategy version of a normal form game is, by definition, a normal form game. Hence, these existence results are valid for equilibrium in mixed strategies as well.
} 


\subsubsection{Existence through Kakutani fixed point theorem}

The following existence result builds on the fact that Nash equilibrium is a special case of minimally altruistic Nash equilibrium (i.e., selfish best-responses). Then, one intuitively expects that the existence of minimally altruistic Nash equilibrium can be guaranteed by taking sufficient conditions for Nash equilibrium (see Nash (1950)) as baseline and adding more conditions or modifying the existing ones. In fact, this is what we do in the following proposition.

We first define $B_{j}^{i}: X \rightarrow X$ such that for every $y \in X$ :

$B_{j}^{i}(y)=\left\{x \in X \mid \forall x_{i}^{\prime} \in X_{i}: u_{j}\left(x_{i}, y_{-i}\right) \geq u_{j}\left(x_{i}^{\prime}, y_{-i}\right)\right\}$.

That is, given a strategy profile of $-i$, agent $j$ chooses a strategy for agent $i$ from $X_{i}$ in order to maximize his own payoff. This correspondence is used in the definition of level-k empathy, which relates the best responses of agents.

Axiom 1 (Level- $k$ Empathy). For $k \geq 2$, agent $j$ is level- $k$ empathetic towards agent $i$ if for every $x \in X: X_{i, k-1}(x) \cap B_{j}^{i}(x)$ is nonempty.

If agent $j$ is empathetic towards agent $i$, then agent $i$ would not complain if agent $j$ selfishly selects a strategy for agent $i$.

Proposition 2. In game $\Gamma_{M A}$, if (i) each $X_{i}$ is a nonempty, compact, and convex subset of a Euclidean space, (ii) each $u_{i}$ is quasiconcave in $x_{j}$ for every $j \in N$ with $i \in S_{j}$ and is continuous in $x$, and (iii) for every $i \in N$, each $j \in S_{i}$ is level- $\varphi(i, j)$ empathetic towards $i$, then MANE exists.

Proof. Take any $i \in N$. It follows from the existence result of Nash equilibrium that $B R_{i}=X_{i, 1}$ is nonempty-valued, convex-valued, and has a closed graph. This implies for all $x \in X$ that $X_{i, 1}(x)$ is nonempty, compact, and convex.

Now, take any $x \in X$ and $i \in N$. Then, take some $j \in S_{i}$ such that $\varphi(i, j)=2$. Since agent $i$ maximizes $u_{j}$ on a nonempty, compact, and convex set, $X_{i, 1}(x)$, and $u_{j}$ is quasiconcave in $x_{i}$ and continuous in $x$ by assumption, it follows that $X_{i, 2}(x)$ is nonempty, compact, and convex. Recursively, for every $x \in X, X_{i,\left|S_{i}\right|}(x)$ is nonempty, compact, and convex as well.

We have that $X_{i,\left|S_{i}\right|}$ is nonempty-valued, convex-valued, and compact-valued. To utilize Kakutani's fixed point theorem we need to show that $X_{i,\left|S_{i}\right|}$ has a closed graph. For that, take any two sequences $\left(x^{m}\right) \rightarrow x$ and $\left(y^{m}\right) \rightarrow y$ such that for every $m$, $x^{m} \in X_{i,\left|S_{i}\right|}\left(y^{m}\right)$. Then, for every $m$, we have $x^{m} \in X_{i, k}\left(y^{m}\right)$ for every $k \in\left\{1, \ldots,\left|S_{i}\right|-1\right\}$. Since for all $m$ and for all $x_{i}^{\prime} \in X_{i}$ : $u_{i}\left(x_{i}^{m}, y_{-i}^{m}\right) \geq u_{i}\left(x_{i}^{\prime}, y_{-i}^{m}\right)$ (by optimality) and $u_{i}$ is continuous, we have $u_{i}\left(x_{i}, y_{-i}\right) \geq u_{i}\left(x_{i}^{\prime}, y_{-i}\right)$ for every $x_{i}^{\prime} \in X_{i}$. Then, $x \in X_{i, 1}(y)$. Now, consider agent $j$ with $\varphi(i, j)=2$. Since $j$ is level-2 empathetic towards $i$ by assumption, we have for all $m$ and for all $x^{\prime} \in X_{i, 1}(y)$ : $u_{j}\left(x_{i}^{m}, y_{-i}^{m}\right) \geq u_{j}\left(x_{i}^{\prime}, y_{-i}^{m}\right)$ by optimality. Then, by continuity of $u_{j}$ in $x$, we also have $u_{j}\left(x_{i}, y_{-i}\right) \geq u_{i}\left(x_{i}^{\prime}, y_{-i}\right)$ for every $x^{\prime} \in X_{i, 1}(y)$, which implies $x \in X_{i, 2}(y)$. Since level- $k$ empathy is assumed for every $j \in S_{i}$, it recursively follows that $x \in X_{i, k}(y)$ for every $k \in\left\{1, \ldots,\left|S_{i}\right|\right\}$. Hence, $x \in X_{i,\left|S_{i}\right|}(y)$, that is $X_{i,\left|S_{i}\right|}$ has a closed graph.

All of these four properties are preserved under finite intersections. Then the joint best response correspondence according to MANE, defined by $\bigcap_{i \in N} X_{i,\left|S_{i}\right|}$, satisfies the conditions of Kakutani's fixed point theorem. Therefore, the set of fixed points is nonempty, i.e. MANE exists.

Note that we just strengthen the quasiconcavity requirement compared to the sufficient conditions for the (standard) existence theorem for Nash equilibrium, and additionally assume level- $k$ empathy. The former is a relatively minor and intuitive modification, whereas the latter is a very restrictive assumption.

\subsubsection{Existence through games with strategic complementarities}

Characterized by increasing joint best reply, games with strategic complementarities (GSC) rely on the extension of Tarski's fixed point theorem for correspondences (see Veinott (1992) and Zhou (1994)) and a lattice-based approach to monotone comparative statics (see Topkis (1978), Vives (1990) and Milgrom and Roberts (1990)). For our minimally altruistic refinement, GSC can be formalized in the following way:

Definition 12. A game $\Gamma_{M A}$ has strategic complementarities à la minimally altruistic Nash (hence, is a GSC à la minimally altruistic Nash) if (i) each $X_{i}$ is a complete lattice, (ii) each best response correspondence according to minimally altruistic Nash equilibrium, $X_{i,\left|S_{i}\right|}$, is nonempty-valued and Veinott-increasing, and (iii) for all $x \in X, X_{i,\left|S_{i}\right|}(x)$ is a subcomplete sublattice of $X$.

The lattice-based approach to monotone comparative statics under the notion of Nash equilibrium establishes that each player's payoff function needs to be supermodular in his own strategies and satisfy increasing differences in order to have Veinott-increasing joint best reply. Stemming from these sufficient conditions on payoffs, such classes of games with monotone best responses are referred to as supermodular games (see Topkis (1998)). However, under the notion of minimally altruistic Nash equilibrium, one needs further requirements to have Veinott-increasing joint best reply: the payoff to each player $i$ needs to be supermodular and satisfy increasing differences with respect to the strategies of every player who cares about $i .^{8}$ In this respect, a minimally altruistic supermodular game can be defined as follows.

Definition 13. A game $\Gamma_{M A}$ is minimally altruistic supermodular if (i) each $X_{i}$ is a nonempty, compact, and complete lattice, (ii) each $u_{i}$ is supermodular in $x_{j}$ for every $j \in N$ with $i \in S_{j}$, (iii) each $u_{i}$ has increasing differences in $\left(x_{j}, x_{-j}\right)$ for every $j \in N$ with $i \in S_{j}$, and (iv) each $u_{i}$ is upper semi-continuous in $x_{j}$ for every $j \in N$ with $i \in S_{j}$.

Note that a minimally altruistic supermodular game is, by definition, a supermodular game. However, a GSC à la minimally altruistic Nash need not be a GSC.

Proposition 3. The following statements on minimally altruistic refinement of Nash equilibrium are valid.

(i) A minimally altruistic supermodular game is a GSC à la minimally altruistic Nash.

(ii) In a GSC à la minimally altruistic Nash (hence, in a minimally altruistic supermodular game), the set of MANE is a nonempty complete lattice.

Proof. For (i), take any $i \in N$ and $j \in S_{i}$ such that $\varphi(i, j)=2$.

First, since $u_{i}$ is upper semi-continuous in $x_{i}, X_{i, 1}(x)=B R_{i}(x)$ is nonempty for all $x \in X$. Then, since $u_{j}$ is upper semi-continuous in $x_{i}, X_{i, 2}(x)$ is also nonempty for all $x \in X$. Similarly, we conclude that for every $k=1, \ldots,\left|S_{i}\right|, X_{i, k}$ is nonempty-valued.

Now, take any $x, y \in X$ with $x<y$. Take $a \in X_{i,\left|S_{i}\right|}(x)$ and $b \in X_{i,\left|S_{i}\right|}(y)$ as well. This implies that $a \in X_{i, k}(x)$ and $b \in X_{i, k}(y)$ for every $k=1, \ldots,\left|S_{i}\right|$. For Veinott-increasingness, we need to show that $a \wedge b \in X_{i,\left|S_{i}\right|}(x)$ and $a \vee b \in X_{i,\left|S_{i}\right|}(y)$. If $a<b$, the result is trivial. If not,

$$
\begin{aligned}
0 & \leq u_{i}\left(a_{i}, x_{-i}\right)-u_{i}\left(a_{i} \wedge b_{i}, x_{-i}\right) \\
& \leq u_{i}\left(a_{i} \vee b_{i}, x_{-i}\right)-u_{i}\left(b_{i}, x_{-i}\right) \\
& \leq u_{i}\left(a_{i} \vee b_{i}, y_{-i}\right)-u_{i}\left(b_{i}, y_{-i}\right) \leq 0 .
\end{aligned}
$$

8 In its essence, this modification is similar to the one we make in Proposition 2. 
Here, the first and the last inequalities follow from optimality since $a \in X_{i, 1}(x)$ and $b \in X_{i, 1}(y)$. Supermodularity implies the second inequality and increasing differences property implies the third inequality. Then, $a \wedge b \in X_{i, 1}(x)$ and $a \vee b \in X_{i, 1}(y)$. Recalling that $a \in X_{i, 2}(x)$ and $b \in X_{i, 2}(y)$, we have

$$
\begin{aligned}
0 & \leq u_{j}\left(a_{i}, x_{-i}\right)-u_{j}\left(a_{i} \wedge b_{i}, x_{-i}\right) \\
& \leq u_{j}\left(a_{i} \vee b_{i}, x_{-i}\right)-u_{j}\left(b_{i}, x_{-i}\right) \\
& \leq u_{j}\left(a_{i} \vee b_{i}, y_{-i}\right)-u_{j}\left(b_{i}, y_{-i}\right) \leq 0 .
\end{aligned}
$$

Here, the first and the last inequalities are valid for every element of $X_{i, 1}(x)$ and $X_{i, 1}(y)$, including $a \wedge b \in X_{i, 1}(x)$ and $a \vee b \in X_{i, 1}(y)$. The second and the third inequalities follow from assumptions that $u_{j}$ is supermodular in $x_{i}$ and $u_{j}$ has increasing differences. Then, $a \wedge b \in X_{i, 2}(x)$ and $a \vee b \in X_{i, 2}(y)$. Similar arguments will follow for every $j^{\prime} \in S_{i}$. Then $a \wedge b \in X_{i,\left|S_{i}\right|}(x)$ and $a \vee b \in X_{i,\left|S_{i}\right|}(y)$, so that $X_{i,\left|S_{i}\right|}$ is Veinott-increasing.

Finally, consider any $x \in X$ and take any $a, b \in X_{i,\left|S_{i}\right|}(x)$. Note that $a, b \in X_{i, k}(x)$ for every $k=1, \ldots,\left|S_{i}\right|$. First we have,

$u_{i}\left(a_{i}, x_{-i}\right)=u_{i}\left(b_{i}, x_{-i}\right) \geq u_{i}\left(c_{i}, x_{-i}\right), \quad \forall c_{i} \in X_{i}$

since $a, b \in X_{i, 1}(x)$ and

$u_{i}\left(a_{i}, x_{-i}\right)+u_{i}\left(b_{i}, x_{-i}\right) \leq u_{i}\left(a_{i} \wedge b_{i}, x_{-i}\right)+u_{i}\left(a_{i} \vee b_{i}, x_{-i}\right)$

by supermodularity of $u_{i}$ in $x_{i}$. Then, it directly follows that $u_{i}\left(a_{i}, x_{-i}\right)=u_{i}\left(a_{i} \wedge b_{i}, x_{-i}\right)=u_{i}\left(a_{i} \vee b_{i}, x_{-i}\right)$, i.e. $a \wedge b$, $a \vee b \in X_{i, 1}(x)$. With this result and similar arguments, we have the following:

$u_{j}\left(a_{i}, x_{-i}\right)=u_{j}\left(b_{i}, x_{-i}\right) \geq u_{j}\left(c_{i}, x_{-i}\right), \quad \forall c_{i} \in X_{i, 1}(x)$

since $a, b \in X_{i, 2}(x)$ and

$u_{j}\left(a_{i}, x_{-i}\right)+u_{j}\left(b_{i}, x_{-i}\right) \leq u_{j}\left(a_{i} \wedge b_{i}, x_{-i}\right)+u_{j}\left(a_{i} \vee b_{i}, x_{-i}\right)$

by supermodularity of $u_{j}$ in $x_{i}$. Similar arguments will follow for every $j^{\prime} \in S_{i}$. Then $a \wedge b \in X_{i,\left|S_{i}\right|}(x)$ and $a \vee b \in X_{i,\left|S_{i}\right|}(x)$, so that $X_{i,\left|S_{i}\right|}(x)$ is a subcomplete sublattice of $X$ for all $x \in X .{ }^{9}$ Then, the game is a GSC à la minimally altruistic Nash.

For (ii), since the properties satisfied by $X_{i,\left|S_{i}\right|}$ are preserved under finite intersections, $\bigcap_{i \in N} X_{i,\left|S_{i}\right|}$ satisfies the conditions of Zhou's fixed point theorem. Therefore, the set of MANE is a nonempty complete lattice.

The utilization of strategic complementarities not only allows us to establish the existence of MANE but also provides a sharp characterization of the set of MANE. Moreover, referring to the constructive proof of Zhou's extension of Tarski's fixed-point theorem to set valued maps (see Echenique (2005)), it provides a simple iterative procedure to compute the extremal equilibria. Note also that we can provide a monotone comparative statics result on the set of MANE, utilizing Topkis' theorem (Topkis, 1998). In particular, letting $T$ be a partially ordered set and $\left(\Gamma_{M A}^{t}\right)_{t \in T}$ be a collection of GSC à la minimally altruistic Nash, the least MANE and the greatest MANE are increasing in $t$ on $T$.

\subsubsection{Existence through Berge-Nash equilibrium}

We first provide a definition of Berge equilibrium $(B E)$. The definition we provide below is commonly referred to as Berge equilibrium in the sense of Zhukovskii (1994) in the literature. ${ }^{10}$ As it can be seen in the definition below, Berge equilibrium has a rather extreme version of altruism embedded in: for any $i$ and

\footnotetext{
9 The best response correspondences are closed-valued in the interval topology (see Zhou (1994)). And a closed interval in a complete lattice $X$ is a subcomplete sublattice of $X$.

10 We refer to Zhukovskii's (1994) definition for Berge equilibrium since Berge (1957) himself offered only an intuitive/informal definition.
}

given player $i$ 's strategy, all other players choose their strategies so as to maximize agent $i$ 's well-being.

Definition 14 (Berge Equilibrium). In game $\Gamma, x^{*}$ is a Berge equilibrium if for every $i \in N$, we have $u_{i}\left(x^{*}\right) \geq u_{i}\left(x_{i}^{*}, x_{-i}\right)$ for every $x_{-i} \in X_{-i}$.

Berge-Nash equilibrium (BNE) directly follows as the intersection of Berge equilibrium with Nash equilibrium.

Definition 15 (Berge-Nash Equilibrium). In game $\Gamma, x^{*}$ is a BergeNash equilibrium if it is both a Berge equilibrium and a Nash equilibrium.

The following definition introduces the reduced game notion we utilize in the existence result that follows.

Definition 16. For given $\Gamma_{M A}, x \in X$ and $i, j \in N$ with $i \neq j$, we define the reduced game $\Gamma_{\{i, j\}}(x)=\left(\{i, j\}, X_{i} \times X_{j},\left(v_{i}, v_{j}\right)\right)$ such that $v_{k}: X_{i} \times X_{j} \rightarrow \mathbb{R}$ is given by $v_{k}\left(a_{i}, a_{j}\right)=u_{k}\left(a_{i}, a_{j}, x_{-\{i, j\}}\right)$ for every $k \in\{i, j\}$ and for every $\left(a_{i}, a_{j}\right) \in X_{i} \times X_{j}$.

Lemma 1. The strategy profile $x^{*} \in \operatorname{MANE}\left(\Gamma_{M A}\right)$ if for every $i, j \in N$ with $j \in S_{i} \backslash\{i\}:\left(x_{i}^{*}, x_{j}^{*}\right) \in B_{i}\left(x_{i}^{*}, x_{j}^{*}\right)$, where $B_{i}(\cdot)$ is a best response of $i$ according to Berge-Nash equilibrium for the reduced game $\Gamma_{\{i, j\}}\left(x^{*}\right)$.

Proof. Take any $x^{*}$ such that for every $i, j \in N$ with $j \in S_{i} \backslash\{i\}$ : $\left(x_{i}^{*}, x_{j}^{*}\right) \in B_{i}\left(x_{i}^{*}, x_{j}^{*}\right)$. Take any $i \in N$. We have $u_{i}\left(x^{*}\right) \geq u_{i}\left(x_{i}, x_{-i}^{*}\right)$, $\forall x_{i} \in X_{i}$. Also, for every $j \in S_{i} \backslash\{i\}: u_{j}\left(x^{*}\right) \geq u_{j}\left(x_{i}, x_{-i}^{*}\right), \forall x_{i} \in X_{i}$. We know that $x^{*} \in X_{i, 1}\left(x^{*}\right)$. Then, recursively, we can say that for every $k \in 2, \ldots,\left|S_{i}\right|, x^{*} \in X_{i, k}\left(x^{*}\right)$. Since $i$ is arbitrarily chosen, the result follows.

The following proposition uncovers an interesting relationship between Berge-Nash equilibrium and minimally altruistic Nash equilibrium.

Proposition 4. If a normal form game $\Gamma$ has a Berge-Nash equilibrium, then $\Gamma_{M A}$ has a minimally altruistic Nash equilibrium. In fact, $\operatorname{BNE}(\Gamma)=\operatorname{BNE}\left(\Gamma_{M A}\right) \subset \operatorname{MANE}\left(\Gamma_{M A}\right)$.

Proof. First of all, BNE of a game equals $B N E$ of the minimally altruistic version of the game, since $B N E$ does not depend on any $S_{i}$ or $\succ_{i}$. For the latter, take $x^{*} \in B N E\left(\Gamma_{M A}\right)$. Consider any relevant reduced game of $\Gamma$ defined as above. Then, obviously, $x^{*}$ satisfies the condition in Lemma 1 for every $i \in N$ and $j \in S_{i} \backslash\{i\}$. Thus, $x^{*} \in \operatorname{MANE}\left(\Gamma_{M A}\right)$.

Larbani and Nessah (2008) provide sufficient conditions for the existence of $B N E$. Under the same set of conditions, the proposition above shows that MANE exists.

\section{Further results}

In this section, we provide further results on minimally altruistic Nash equilibrium.

The following remark relates MANE to NE and BNE. It focuses on the trivial case where players care only about themselves. In that case, MANE and NE are, not surprisingly, equivalent. On the other hand, the same remark also states that even if each player cares about all the other players, MANE may not be reduced to BNE.

Remark 1. In a game $\Gamma_{M A}$, if for every $i \in N, S_{i}=\{i\}$, then $\operatorname{MANE}\left(\Gamma_{M A}\right)=N E\left(\Gamma_{M A}\right)$. On the other hand, even if for all $i \in N$, $S_{i}=N$, it may be the case that $\operatorname{MANE}\left(\Gamma_{M A}\right) \neq B N E\left(\Gamma_{M A}\right)$. 
Proof. In the former case, for all $i \in N, X_{i,\left|S_{i}\right|}=X_{i, 1}=B R_{i}$. The result follows. For the latter case, we again provide an example. Consider the following normal form game with three players in which Player 1 has two strategies whereas Player 2 and Player 3 have only one strategy.

$$
\begin{array}{lc} 
& \multicolumn{1}{c}{x_{3}} \\
& x_{2} \\
x_{1} & 2,1,0 \\
y_{1} & 2,0,1 \\
\hline
\end{array}
$$

Here, the set of $B N E$ is empty but for every $\left(S_{i}\right)_{i \in N}$ and $\left(\succ_{i}\right)_{i \in N}$, MANE exists.

The following remark states that the set of MANE will be smaller as for a given player $i,\left|S_{i}\right|$ gets larger with a specific modification on the strict order, $\succ_{i}$. In particular, the remark indicates that if a player $i$, in addition to the current set of players he cares about, starts to care about some other players who he did not care about initially, the set of MANE of this new game will not be larger than the MANE of the initial game.

Remark 2. Let $\Gamma$ be a normal form game. Take an arbitrary $i \in N$, and define $\succ_{i}^{\prime}$ on $N$. Let $\Gamma_{M A}^{k}$ with $\left(S_{i, k}, \succ_{i, k}\right)$ be such that $S_{i, k}$ consists of the top $k$ elements in $\succ_{i}^{\prime}$ and $\succ_{i, k}=\left.\succ_{i}^{\prime}\right|_{s_{i, k}}$ for given $\left(S_{j}\right)_{j \in N \backslash\{i\}}$ and $\left(\succ_{j}\right)_{j \in N \backslash\{i\}}$. Then, $\operatorname{MANE}\left(\Gamma_{M A}^{\beta}\right) \subset \operatorname{MANE}\left(\Gamma_{M A}^{\alpha}\right)$ if $\alpha<\beta$.

Proof. Take $x^{*} \in \operatorname{MANE}\left(\Gamma_{M A}^{\beta}\right)$ for some $\beta \in\{2, \ldots,|N|\}$. Consider an arbitrary $\alpha$ with $\alpha<\beta$. By definition, for every $i \in N$ and $k \in\{1, \ldots, \beta\}, x^{*} \in X_{i, k}\left(x^{*}\right)$. Since $\alpha<\beta$, it trivially follows that $x^{*} \in \operatorname{MANE}\left(\Gamma_{M A}^{\alpha}\right)$.

The next remark is, in fact, a corollary to the remark above. It implies that starting with completely selfish players and increasing the cardinality of their priority sets in the way described in Remark 2, MANE refines the set of $N E$ in a monotonic fashion.

Remark 3. Let $\left(\Gamma_{M A}^{k}\right)=\left(\left(N,\left(X_{i}\right)_{i \in N},\left(S_{i}^{k}\right)_{i \in N},\left(\succ_{i}^{k}\right)_{i \in N},\left(u_{i}\right)_{i \in N}\right)\right)$ be a collection of minimally altruistic versions of the same normal form game $\Gamma$. Let $S_{i}^{0}=\{i\}$ for every $i \in N$ and define $\Gamma_{M A}^{k+1}$ by a modification on $\Gamma_{M A}^{k}$ for some $i \in N$ such that $\sum_{i \in N}\left|S_{i}^{k+1} \backslash\{i\}\right|=$ $\sum_{i \in N}\left|S_{i}^{k} \backslash\{i\}\right|+1=k+1$. Then, the cardinality of the set of MANE is nonincreasing in $k$.

Proof. The result follows from a recursive application of the exercise described in Remark 2.

The common message of the following remarks (Remarks 49) is that the set of MANE is, as expected, very sensitive to each player's priority set and the priority orders on these sets. Remarks here show that even very minor changes on priority sets and orders may lead to changes in the set of equilibria in ways that cannot be systematically predicted. ${ }^{11}$

Remark 4. Let $\Gamma_{M A}$ and $\Gamma_{M A^{\prime}}$ be minimally altruistic versions of the same normal form game $\Gamma$ such that the only difference is that for some $i \in N, S_{i}^{\prime} \subset S_{i}$ and $\succ_{i}^{\prime}=\left.\succ_{i}\right|_{S_{i}^{\prime}}$. Then, it may be the case that $\operatorname{MANE}\left(\Gamma_{M A}\right) \not \subset \operatorname{MANE}\left(\Gamma_{M A^{\prime}}\right)$ and $\operatorname{MANE}\left(\Gamma_{M A^{\prime}}\right) \not \subset \operatorname{MANE}\left(\Gamma_{M A}\right)$.

\footnotetext{
11 The sensitivity of the predictions of our refinement concept to priority sets and orders should not be seen as a weakness. This sensitivity is of the same sort Nash equilibrium has with respect to payoffs. It is a known fact that improving the exante position of a player in a game by, for instance, increasing his payoffs in some strategy combinations does not necessarily imply a higher equilibrium payoff for the player.
}

Proof. Consider the following normal form game:

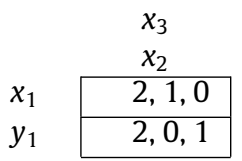

Let $S_{1}=\{1,2,3\}, S_{1}^{\prime}=\{1,3\}$, and $1 \succ_{1} 2 \succ_{1} 3$. Define $\Gamma_{M A}$ and $\Gamma_{M A^{\prime}}$ accordingly. Now, $\operatorname{MANE}\left(\Gamma_{M A}\right)=\left\{\left(x_{1}, x_{2}, x_{3}\right)\right\}$ and $\operatorname{MANE}\left(\Gamma_{M A^{\prime}}\right)=\left\{\left(y_{1}, x_{2}, x_{3}\right)\right\}$.

Remark 5. Let $\Gamma_{M A}$ and $\Gamma_{M A^{\prime}}$ be minimally altruistic versions of the same normal form game $\Gamma$ such that the only difference is that for some $i \in N, \succ_{i} \neq \succ_{i}^{\prime}$. Then, it may be the case that $\operatorname{MANE}\left(\Gamma_{M A}\right) \not \subset \operatorname{MANE}\left(\Gamma_{M A^{\prime}}\right)$ and $\operatorname{MANE}\left(\Gamma_{M A^{\prime}}\right) \not \subset \operatorname{MANE}\left(\Gamma_{M A}\right)$.

Proof. Consider the following normal form game:

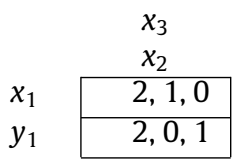

Let $S_{1}=\{1,2,3\}, 1 \succ_{1} 2 \succ_{1} 3$ and $1 \succ_{1}^{\prime} 3 \succ_{1}^{\prime} 2$. Define $\Gamma_{M A}$ and $\Gamma_{M A^{\prime}}$ accordingly. Now, $\operatorname{MANE}\left(\Gamma_{M A}\right)=\left\{\left(x_{1}, x_{2}, x_{3}\right)\right\}$ and $\operatorname{MANE}\left(\Gamma_{M A^{\prime}}\right)=\left\{\left(y_{1}, x_{2}, x_{3}\right)\right\}$.

Remark 6. Let $\Gamma_{M A}$ and $\Gamma_{M A^{\prime}}$ be minimally altruistic versions of the same normal form game $\Gamma$ such that the only difference is that for some $i \in N, S_{i}^{\prime} \backslash S_{i}=\left\{j^{\prime}\right\}$ and $S_{i} \backslash S_{i}^{\prime}=\{j\}$ with $\varphi(i, j)=\varphi^{\prime}\left(i, j^{\prime}\right)$. Then, it may be the case that $\operatorname{MANE}\left(\Gamma_{M A}\right) \not \subset \operatorname{MANE}\left(\Gamma_{M A^{\prime}}\right)$ and $\operatorname{MANE}\left(\Gamma_{M A^{\prime}}\right) \not \subset \operatorname{MANE}\left(\Gamma_{M A}\right)$.

Proof. Consider the following normal form game:

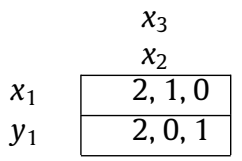

Let $S_{1}=\{1,2\}$ and $S_{1}^{\prime}=\{1,3\}$. Define $\Gamma_{M A}$ and $\Gamma_{M A^{\prime}}$ accordingly. Now, $\operatorname{MANE}\left(\Gamma_{M A}\right)=\left\{\left(x_{1}, x_{2}, x_{3}\right)\right\}$ and $\operatorname{MANE}\left(\Gamma_{M A^{\prime}}\right)=$ $\left\{\left(y_{1}, x_{2}, x_{3}\right)\right\}$.

Remark 7. Let $\Gamma_{M A}$ and $\Gamma_{M A^{\prime}}$ be minimally altruistic versions of the same normal form game $\Gamma$ such that the only difference is that for some $i, j \in N$ with $i \notin S_{j} \cup S_{j}^{\prime}$ and $j \notin S_{i} \cup S_{i}^{\prime}: \varphi(i, k)=\varphi^{\prime}(j, k)$ and $\varphi(j, k)=\varphi^{\prime}(i, k)$ for every $k \in N \backslash\{i, j\}$. Then, it may be the case that $\operatorname{MANE}\left(\Gamma_{M A}\right) \not \subset \operatorname{MANE}\left(\Gamma_{M A^{\prime}}\right)$ and $\operatorname{MANE}\left(\Gamma_{M A^{\prime}}\right) \not \subset \operatorname{MANE}\left(\Gamma_{M A}\right)$.

Proof. Consider the following normal form game:

\begin{tabular}{ll}
\multicolumn{1}{c}{} & $x_{3}$ \\
\multicolumn{1}{c}{} & $x_{2}$ \\
$x_{1}$ & $2,1,0$ \\
$y_{1}$ & $2,0,1$ \\
\hline
\end{tabular}

Let $S_{1}=\{1,2\}, S_{3}=\{3\}, S_{1}^{\prime}=\{1\}$ and $S_{3}^{\prime}=\{3,2\}$. Define $\Gamma_{M A}$ and $\Gamma_{M A^{\prime}}$ accordingly. Now, $\operatorname{MANE}\left(\Gamma_{M A}\right)=\left\{\left(x_{1}, x_{2}, x_{3}\right)\right\}$ and $\operatorname{MANE}\left(\Gamma_{M A^{\prime}}\right)=\left\{\left(x_{1}, x_{2}, x_{3}\right),\left(y_{1}, x_{2}, x_{3}\right)\right\}$. Hence, $\operatorname{MANE}\left(\Gamma_{M A}\right) \not \subset$ $\operatorname{MANE}\left(\Gamma_{M A^{\prime}}\right)$. Note that the converse is symmetric.

Remark 8. Let $\Gamma_{M A}$ and $\Gamma_{M A^{\prime}}$ be minimally altruistic versions of the same normal form game $\Gamma$ such that the only difference is that for some $j \in N$ and for every $i \in N \backslash\{j\}: \varphi(i, j)>\varphi^{\prime}(i, j)$. Then, it may be the case that maximum equilibrium payoff for player $j$ in $\operatorname{MANE}\left(\Gamma_{M A^{\prime}}\right)$ is smaller than minimum equilibrium payoff for player $j$ in $\operatorname{MANE}\left(\Gamma_{M A}\right)$. 
Proof. Consider the following normal form game:

\begin{tabular}{l|c|c|}
\multicolumn{2}{c}{$x_{2}$} & \multicolumn{1}{c}{$x_{3}$} \\
\cline { 2 - 3 }$x_{1}$ & $1,3,0$ & $1,4,0$ \\
\cline { 2 - 3 }$y_{1}$ & $1,2,1$ & $2,0,1$ \\
\cline { 2 - 3 }$z_{1}$ & $0,0,0$ & $2,1,0$ \\
\cline { 2 - 3 } & &
\end{tabular}

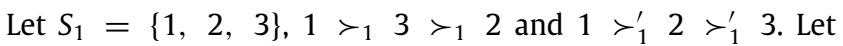
$S_{3}=\{1,2,3\}, 3 \succ_{3} 1 \succ_{3} 2$ and $3 \succ_{3}^{\prime} 2 \succ_{3}^{\prime} 1$. Define $\Gamma_{M A}$ and $\Gamma_{M A^{\prime}}$ accordingly. Now, for $\Gamma_{M A^{\prime}}$, there is a unique MANE, $\left(z_{1}, y_{2}, x_{3}\right)$, which yields 1 to Player 2. For $\Gamma_{M A}$, there is also a unique MANE, $\left(y_{1}, x_{2}, x_{3}\right)$, which yields 2 to Player 2 .

Remark 9. Let $\Gamma_{M A}$ and $\Gamma_{M A^{\prime}}$ be minimally altruistic versions of the same normal form game $\Gamma$ such that the only difference is that for some $i \in N, S_{i}^{\prime} \backslash S_{i}=\{j\}$ and $\succ_{i}=\left.\succ_{i}^{\prime}\right|_{S_{i}}$. Then, it may be the case that an equilibrium that yields player $i$ the highest (the lowest) payoff is eliminated.

Proof. Consider the following normal form game:

\begin{tabular}{l|l|l|} 
& \multicolumn{1}{c}{$x_{2}$} & \multicolumn{1}{c}{$y_{2}$} \\
\cline { 3 - 3 }$x_{1}$ & $a, 2$ & 1,3 \\
\cline { 2 - 3 }$y_{1}$ & $a, 1$ & 2,0 \\
\cline { 2 - 3 }$z_{1}$ & 0,0 & 2,1 \\
\cline { 2 - 3 } & &
\end{tabular}

Let $S_{1}=\{1\}, S_{1}^{\prime}=\{1,2\}$ and $S_{2}=S_{2}^{\prime}=\{2\}$. Define $\Gamma_{M A}$ and $\Gamma_{M A^{\prime}}$ accordingly. Now, for $\Gamma_{M A}$, the set of MANE is $\left\{\left(y_{1}, x_{2}\right)\right.$, $\left.\left(z_{1}, y_{2}\right)\right\}$. For $\Gamma_{M A^{\prime}},\left(z_{1}, y_{2}\right)$ is the unique MANE if $a>0$. Therefore, if $a>2(a<2)$, then the equilibrium that yields Player 1 the highest (the lowest) payoff is eliminated.

The remark below states that if each and every player in a game has a unique best response to some $N E$, then that $N E$ is also a MANE.

Remark 10. If $x^{*}$ is a NE in which $B R_{i}\left(x^{*}\right)=\left\{\left(x_{i}^{*}, \cdot\right)\right\}$ for every $i \in N$, then $x^{*}$ is also a MANE.

Proof. Take some $N E, x^{*}$, in which $B R_{i}\left(x^{*}\right)=\left\{\left(x_{i}^{*}, \cdot\right)\right\}$ for every $i \in$ $N$. Take any $i \in N$. Regardless of $S_{i}$ and $\succ_{i}, X_{i, k}\left(x^{*}\right)=\left\{\left(x_{i}^{*}, \cdot\right)\right\}$ for every $k \in\left\{1, \ldots,\left|S_{i}\right|\right\}$. Since $i$ is arbitrary, the result follows.

Finally, the following remark shows that in some games MANE and $N E$ are not different. In particular, if for each player in the game, any two different strategies always give different payoffs, then the $M A N E$ of this game will be equivalent to the NE of the game.

Remark 11. In a game $\Gamma_{M A}$, if $\forall i \in N, \forall x_{-i} \in X_{-i}$, and $\forall x_{i}, y_{i} \in X_{i}$ : $u_{i}\left(x_{i}, x_{-i}\right) \neq u_{i}\left(y_{i}, x_{-i}\right)$, then $\operatorname{MANE}\left(\Gamma_{M A}\right)=N E\left(\Gamma_{M A}\right)$.

Proof. Any $N E$ of $\Gamma_{M A}$ satisfies the condition in Remark 10, hence $N E\left(\Gamma_{M A}\right) \subset \operatorname{MANE}\left(\Gamma_{M A}\right)$. The converse is true since MANE is a refinement of $N E$.

\section{Examples}

In this section, we provide examples where minimally altruistic refinement leads to significantly different predictions than Nash equilibrium.

The following example shows an instance where a player with a unique strategy can influence the set of minimally altruistic Nash equilibria. We know that this cannot happen when Nash equilibrium is employed. Moreover, in this example, $N E$ gives practically uninformative predictions whereas MANE makes a sharp prediction about the outcome of the game.
Example 1. Consider the normal form game where $N=\{1,2,3\}$, first and second players have two strategies and the third player has only one strategy. Obviously, the third player has no influence on the determination of $N E$. In contrast, we show that he (indirectly) influences the set of MANE.

\begin{tabular}{l|c|c|} 
& \multicolumn{2}{c}{$x_{3}$} \\
& $x_{2}$ & $y_{2}$ \\
$x_{1}$ & $3,0,5$ & $4,0,4$ \\
\cline { 2 - 3 }$y_{1}$ & $3,4,0$ & $4,4,4$ \\
\cline { 2 - 3 } & &
\end{tabular}

Here, any strategy profile is a pure strategy NE. Note that this game is a minimally altruistic supermodular game given that $x_{i}>$ $y_{i}$ for every $i \in\{1,2\}, S_{1}=\{1,2\}$, and $S_{2}=\{2,3\}$. We have the set of pure strategy MANE as $\left\{\left(y_{1}, y_{2}, x_{3}\right)\right\}$. Thus, the equilibrium set is refined to a singleton.

The following example shows an instance where, again, any strategy profile is a NE. On the other extreme, neither $B E$ nor $B N E$ exists in this game. Nevertheless, MANE exists and moreover it is unique.

Example 2. Consider the normal form game where $N=\{1,2,3\}$ and set $X_{i}=\left\{x_{i}, y_{i}, z_{i}\right\}$ for every $i \in N$. Let $S_{1}=\{1,2\}, S_{2}=\{2,3\}$, and $S_{3}=\{3,1\}$. Let $u_{1}(\theta)=2$ if $\theta_{-1}=\left(x_{2}, x_{3}\right), u_{1}(\theta)=1$ if $\theta_{-1}=\left(x_{2}, \cdot\right)$ or $\theta_{-1}=\left(\cdot, x_{3}\right)$ but not $\theta_{-1}=\left(x_{2}, x_{3}\right)$, and $u_{1}(\theta)=0$ otherwise. Define $u_{2}$ and $u_{3}$ similarly using $y_{j}$ and $z_{j}$, respectively. Note that player $i$ cannot affect his own payoff in this game. Also note that this game is a GSC à la minimally altruistic Nash for any orders defined on $X_{i}$ 's. It is easy to see that in this game, any strategy profile is a pure strategy NE. Moreover, there exists no BE. Hence, there exists no $B N E$ either. However, there is a unique pure strategy MANE, which is $\left(y_{1}, z_{2}, x_{3}\right)$. $\diamond$

In the divide-the-dollar game (a special case of the Nash demand game where the bargaining frontier is linear), using $N E$ does not give sharp predictions. In fact, there are infinitely many Nash equilibria of this game: any point on the bargaining frontier is a $N E$. In the literature on such problems, researchers usually modify the rules of the game such that the equilibrium set is a singleton. ${ }^{12}$ In the following example, we also modify this game, but in a way that the set of $N E$ remains unchanged yet MANE refines NE significantly.

Example 3 (Pie Division Game). Consider the simple pie division game (usually called 'divide the dollar' game) in which every player $i \in N$ simultaneously claims $c_{i} \in[0,1]$ of a pie of size 1 . If $\sum_{i \in N} c_{i}>1$, then each player receives 0 and if $\sum_{i \in N} c_{i} \leq 1$, then each player $i$ receives his claim, $c_{i} \cdot{ }^{13}$ We modify this game as follows: (i) (efficiency) if $\sum_{i \in N} c_{i}<1$ then $1-\sum_{i \in N} c_{i}$ is equally divided between players, i.e. player $i$ receives $c_{i}+k$ where $k=\left(1-\sum_{i \in N} c_{i}\right) / n$, and (ii) (satiation) each player is indifferent between getting more than $3 / 4$ and getting $3 / 4 .{ }^{14}$ In this modified version, any strategy profile $\left(c_{i}\right)_{i \in N}$ such that $\sum_{i \in N} c_{i}=1$ is still a $N E$ (as it is in the standard version). However, MANE refines the set of $N E$ to

$\left\{\left(c_{i}\right)_{i \in N} \mid \sum_{i \in N} c_{i}=1\right.$ and $\forall i \in N$ with $\left.S_{i} \backslash\{i\} \neq \varnothing: c_{i} \leq 3 / 4\right\}$.

\footnotetext{
12 For this approach, the reader is referred to Brams and Taylor (1994), Anbarc1 (2001), Ashlagi et al. (2012) and Cetemen and Karagözoğlu (2013).

13 In this standard version of the game, the strategy profile with $\forall i \in N: c_{i}=1$ is a $N E$, but it is not a MANE. In the modified version, the set of equilibria is refined further.

14 This can be justified in a setting where nobody is able to consume more than $3 / 4$ (e.g., the pie will go bad before a single person can eat the three quarters of it) Alternatively, one can also think about consumption capacities.
} 
In fact, if everyone cares about someone else and every player is indifferent between receiving more than $1 / n$ and receiving $1 / n$, then there would be a unique pure strategy MANE. Note that the set of $N E$ would still not change.

The following example is a version of the well-known threshold public good game. It shows that if players in this game care about each other's well-being, MANE gives a different prediction than $N E$, and in a special case, MANE predicts that a socially optimal level of public good will be produced whereas some $N E$ still predict a suboptimal level of public good production.

Example 4 (Threshold Public Good Game). In this game, players contribute some amount, $c_{i} \in[0, C]$, to cooperatively produce two public goods, labeled $k$ and $m$. If $\sum_{i \in N} c_{i}<K$, then no public good is produced. Thus, each player receives 0 , but incurs the cost $c_{i}$, leading to a net payoff of $-c_{i}$. If $\sum_{i \in N} c_{i} \geq K$ but $\sum_{i \in N} c_{i}<M$, then $k$ will be produced but not $m$. Thus, each player receives his value $v_{i, k}<K$ and incurs the cost $c_{i}$, leading to a net payoff of $v_{i, k}-c_{i}$. Besides, the residual amount, $\sum_{i \in N} c_{i}-K$, is realized as additional payoff by some players. In particular, assume that there are two types of players which differ in these additional utilities. Type $t_{m}$ agents realize $\pi\left(t_{m}\right)=0$ in case $\sum_{i \in N} c_{i}<M$ and they realize $\pi\left(t_{m}\right)=\sum_{i \in N} c_{i}-K$ in case $\sum_{i \in N} c_{i} \geq M$; whereas type $t_{k}$ agents realize $\pi\left(t_{k}\right)=\sum_{i \in N} c_{i}-K$ as long as $\sum_{i \in N} c_{i} \geq K$. In this game, if $\sum_{i \in N} v_{i, k}<K$, then public good $k$ cannot be produced in equilibrium since $c_{i}=0$ for each $i$. If not, letting $C \geq M$, in some pure strategy $N E$, public good $m$ cannot be produced. However, in all pure strategy MANE except the one with $\forall i \in N: c_{i}=0$, both public goods are produced under the condition that at least one type $t_{k}$ player cares about some type $t_{m}$ player. The special case in which the optimal production, $c_{i}=C$ for every $i \in N$, will be realized is the case where every player cares about some type $t_{m}$ agent. $\diamond$

Finally, we study a simple version of the famous congestion game (Rosenthal, 1973). In the following example, six out of eight possible strategy profiles are $N E$, whereas with the given priority structure, only two of them are MANE.

Example 5 (Congestion Game). In this game, the set of players is $N=\{1,2,3\}$. There are two possible routes from their originating point to a destination, labeled $A$ and $B$. Players simultaneously choose a route. Hence, the strategy set is $X_{i}=\{A, B\}$ for every $i \in N$. If a route is used by more than one player, there will be a congestion. In that case, none of these players using the same route can reach the destination on time. If a player can reach the destination on time, he receives a payoff of 1 . Otherwise, he receives a payoff of 0 . Clearly, there are six pure strategy $N E$ of this game: $(A, B, B)$, $(A, B, A),(B, A, A),(B, A, B),(B, B, A)$, and $(A, A, B)$. Now, let us assume that Player 3 is in an emergency situation and hence both Player 1 and Player 2 care about Player 3 in a minimally altruistic sense. Moreover, Player 3 is still assumed to be selfish. It follows that the set of pure strategy MANE is $\{(A, A, B),(B, B, A)\}$. Hence, even if Player 1 and 2 cared about Player 3 in a minimal fashion, this leads to the elimination of $N E$ that were favorable to them. Moreover, note that neither Berge equilibrium nor Berge-Nash equilibrium exists in this game.

\section{Conclusion}

In this paper, we first introduce a minimal yet very reasonable notion of altruism. It is minimal since it stipulates that people care about others only after maximizing their own well-being. ${ }^{15}$ Then, we use this notion to refine the set of Nash equilibria in normal

\footnotetext{
15 Despite the fact that we focus on altruism in this paper, the scope of the paper could be more general than altruism. In particular, it could concern games where each player's preferences over outcomes are lexicographic over a finite collection of utility functions. We would like to thank an anonymous reviewer for pointing this out.
}

form games. We prove the existence of equilibrium under this new refinement through three different channels, relate it to Nash equilibrium and Berge-Nash equilibrium, and provide examples showing the usefulness of this refinement concept. The definition of minimally altruistic Nash equilibrium contains Nash equilibrium as a special case (e.g., selfish best-responses). It gives sharper and more reasonable predictions than Nash equilibrium if players not only care about themselves but also care about other players' well-being in a lexicographic fashion when best-responding to others' strategies in a strategic game. Minimally altruistic Nash equilibrium shares a flavor similar to that of Berge-Nash equilibrium in that it is also based on altruistic behavior. However, from an empirical point of view we believe that it is much easier to support minimal altruism. ${ }^{16}$ Moreover, from a theoretical perspective, there are games where Berge-Nash equilibrium (or Berge equilibrium) does not exist whereas minimally altruistic Nash equilibrium exists. Future theoretical work may study simultaneous application of the concept together with other refinement concepts, investigate general families of games where its use is fruitful, and characterize the family of games where the effects of changes in priority structure on equilibrium payoffs is more systematic. The investigation of the relationship (e.g., the domain of games where they coincide) between minimally altruistic Nash equilibrium and Kantian equilibrium (see Roemer (2010)) may also be of interest. Finally, future experimental work may shed light on the empirical validity of its predictions.

\section{Acknowledgments}

We thank two anonymous reviewers, Gary Bolton, Nikolaos Georgantzis, Werner Güth, İsa Emin Hafalır, Kevin Hasker, Tarık Kara, Özgür Kıbrıs, Semih Koray, Jean Laine, Jean-François Laslier, Arno Riedl, Ariel Rubinstein, and Walter Trockel for helpful comments and discussions. The usual disclaimers apply.

\section{References}

Anbarc1, N., 2001. Divide-the-dollar game revisited. Theory and Decision 50 (4), 295-304.

Ashlagi, I., Karagözoğlu, E., Klaus, B., 2012. A non-cooperative support for equal division in estate division problems. Mathematical Social Sciences 63 (3), 228-233.

Berge, C., 1957. Théorie Générale des Jeux á n-Personnes. Gauthier Villars, Paris

Bolton, G.E., Ockenfels, A., 2000. ERC: A theory of equity, reciprocity, and competition. American Economic Review 90 (1), 166-193.

Brams, S.J., Taylor, A.E., 1994. Divide the dollar: Three solutions and extensions. Theory and Decision 37 (2), 211-231.

Cappelen, A., Fest, S., Sørensen, E., Tungodden, B., 2013. Risk taking and overattribution of individual responsibility. NHH Working Paper.

Cetemen, E.D., Karagözoğlu, E., 2013. Implementing equal division with an ultimatum threat. Theory and Decision. http://dx.doi.org/10.1007/s11238-0139394-Z.

Charness, G., Rabin, M., 2002. Understanding social preferences with simple tests. Quarterly Journal of Economics 117 (3), 817-869.

Cox, J., Friedman, D., Gjerstad, S., 2007. A tractable model of reciprocity and fairness. Games and Economic Behavior 59 (1), 17-45.

Cox, J., Friedman, D., Sadiraj, V., 2008. Revealed altruism. Econometrica 76 (1),

Dogan, B., 2013. Eliciting the socially optimal allocation from responsible agents. Mimeo, Rochester University.

Dutta, B., Laslier, J.-F., 2010. Costless honesty in voting. In: 10th International Meeting of the Society for Social Choice and Welfare, Moscow.

Dutta, B., Sen, A., 2012. Nash implementation with partially honest individuals. Games and Economic Behavior 74 (1), 154-169.

Echenique, F., 2005. A short and constructive proof of Tarski's fixed-point theorem. International Journal of Game Theory 33 (2), 215-218.

Engelmann, D., Strobel, M., 2004. Inequality aversion, efficiency, and maximin preferences in simple distribution experiments. American Economic Review 94 (4), 857-869.

Falk, A., Fischbacher, U., 2006. A theory of reciprocity. Games and Economic Behavior 54 (2), 293-315.

Fehr, E., Fischbacher, U., 2003. The nature of human altruism. Nature 425, 785-791.

\footnotetext{
16 See Fehr and Fischbacher (2003) and the references therein for partial support from experimental work, which shows that as the cost of altruism decreases altruistic behavior is more frequently observed.
} 
430

E. Karagözoğlu et al. / Mathematical Social Sciences 66 (2013) 422-430

Fehr, E., Gächter, S., 2002. Altruistic punishment in humans. Science 415, 137-140. Fehr, E., Schmidt, K.M., 1999. A theory of fairness, competition, and cooperation. Quarterly Journal of Economics 114 (3), 817-868.

Fishkin, J.S., 1982. Limits of Obligation. Yale University Press, New Haven.

Forsythe, R., Horowitz, J., Gavin, N.E., Sefton, M., 1994. Fairness in simple bargaining experiments. Games and Economic Behavior 6 (3), 347-369.

Gintis, H., Bowles, S., Boyd, R., Feer, E., 2003. Explaining altruistic behavior in humans. Evolution and Human Behavior 24 (3), 153-172.

Güth, W., Levati, M.V., Ploner, M., 2012. An experimental study of the generosity game. Theory and Decision 72 (1), 51-63.

Güth, W., Pull, K., Stadler, M., Stribeck, A., 2010. Equity versus efficiency? Evidence from three-person generosity experiments. Games 1 (2), 89-102.

Güth, W., Schmittberger, R., Schwarze, B., 1982. An experimental analysis of ultimatum bargaining. Journal of Economic Behavior and Organization 3 (4), $367-388$.

Güth, W., Van Lame, E., 1998. Information, strategic behavior and fairness in ultimatum bargaining: An experimental study. Journal of Mathematical Psychology 42 (2), 227-247.

Hardin, G., 1977. The Limits of Altruism. Indiana University Press, Bloomington.

Kakutani, S., 1941. A generalization of Brouwer's fixed point theorem. Duke Mathematical Journal 8 (3), 457-459.

Knoblauch, V., 2001. Is altruism feasible? Interdependent preferences provide the answer. University of Connecticut Working Paper Series \#2001-04.

Larbani, M., Nessah, R., 2008. A note on the existence of Berge and Berge-Nash equilibrial. Mathematical Social Sciences 55 (2), 258-271.

Laslier, J.-F., Weibull, J., 2013. An incentive-compatible Condorcet jury theorem. Scandinavian Journal of Economics 115 (1), 84-108.
Levine, D.K., 1998. Modeling altruism and spitefulness in experiments. Review of Economic Dynamics 1 (3), 593-622.

Milgrom, P., Roberts, J., 1990. Rationalizability, learning, and equilibrium in games with strategic complementarities. Econometric 58 (6), 1255-1277.

Nash, J.F., 1950. Equilibrium points in $n$-person games. Proceedings of the National Academy of Sciences 36 (1), 48-49.

Rabin, M., 1993. Incorporating fairness into game theory and economics. American Economic Review 83 (5), 1281-1302.

Roemer, J.E., 2010. Kantian equilibrium. Scandinavian Journal of Economics 112 (1), $1-24$.

Rosenthal, R.W., 1973. A class of games possessing pure-strategy Nash equilibrial. International Journal of Game Theory 2 (1), 65-67.

Roth, A.E., Prasnikar, V., Okuno-Fujiwara, M., Zamir, S., 1991. Bargaining and market behavior in Jerusalem, Ljubljana, Pittsburgh and Tokyo. American Economic Review 81 (5), 1068-1095.

Topkis, D.M., 1978. Minimizing a submodular function on a lattice. Operations Research 26 (2), 305-321.

Topkis, D.M., 1998. Supermodularity and Complementarity. Princeton University Press, New Jersey.

Veinott, A., 1992. Lattice Programming: Qualitative Optimization and Equilibrial. Mimeo, Stanford University.

Vines, X., 1990. Nash equilibrium with strategic complementarities. Journal of Mathematical Economics 19 (3), 305-321.

Chou, L., 1994. The set of nash equilibria of a supermodular game is a complete lattice. Games and Economic Behavior 7, 295-300.

Zhukovskii, V.I., 1994. Linear Quadratic Differential Games. Naoukova Doumka, Kiev. 